\title{
Channel Swapping based Dynamic Channel Allocation
}

\author{
$G$ Vijay Kumar and $A P$ Shivaprasad \\ Department of Electrical Communication Engineering, \\ Indian Institute of Science, \\ Bangalore 560012. \\ vijayg@protocol.ece.iisc.ernet.in and aps@ece.iisc.ernet.in
}

\begin{abstract}
Several DCA schemes have been proposed to improve the quality and carried traffic in mobile cellular radio system [6-8]. Most of these schemes assign channels to calls based on the constraint imposed by cochannel interference, and ignore the ACI (Adjacent Channel Interference) constraint. Incorporating the ACI constraint results in increasing the blocking probability. In order to overcome this problem we propose a new channel allocation scheme based on the minimum cost function and "Channel Swapping" idea. The scheme involves, swapping the assigned channels from the interfering cells to assign a channel for a call request which is about to fail. Performance evaluation of the proposed scheme is carried out by computer simulation in terms of carried traffic and call blocking, and compared with the DCA schemes of $[1],[2]$, and [3].
\end{abstract}

\section{Introduction}

Technological advances and fast development of hand held wireless terminals have facilitated the rapid growth of wireless communications and mobile computing. The tremendous growth of the wireless/mobile user population, coupled with the bandwidth requirements of multimedia applications, requires efficient reuse of the radio spectrum allocated to wireless/mobile communications.

The basic prohibiting factor in radio spectrum reuse is interference caused by the environment or other mobiles. There are two types of interferences (i) Cochannel interference and (ii) Noncochannel interference. Frequency reuse implies that in a given coverage area there are several cells that use the same set of frequencies, and the interference between signals from these cells is called "cochannel interference". "Adjacent channel interference (ACI)" is a broad term which comes under the category of noncochannel interference. Interference resulting from signals which are adjacent in frequency to the desired signal is called "adjacent channel interference". ACI results from imperfect receiver filters which allow nearby frequencies to leak into the passband.

In addition to Cochannel Interference (CI) constrain, the design of a wireless cellular system must also include measures to limit adjacent channel interference (ACI). Channel impairments such as crosstalk, premature hand-offs, and dropped calls may result from ACI, leading to degradation of quality of service. Although channel filters in both the base station and the mobile unit receivers significantly attenuate signal from adjacent channels [9], [10], severe interference may occur in circumstances where the received signal level of an adjacent channel greatly exceeds that of the desired channel. This situation arises often in mobile cellular environments due to the distance difference between the mobile units and the base stations. ACI can be reduced by frequency assignment plan such that no two adjacent frequencies are assigned to the same cell.

By incorporating the ACI constraint, the quality of the system increases but at the same time the call blocking probability is increased for a given offered traffic. In this paper a new Channel Swapping based Dynamic Channel Allocation (CS-DCA) scheme is proposed to decrease the call blocking probability. Further the CS-DCA technique proposed here improves the performance when compared to other methods which incorporate the ACI constraint.

\section{Adjacent Channel Interfer- ence}

ACI can be eliminated on the basis of the channel assignment, the filter characteristics, and the reduction of near-end-far-end interference. Assume the worst case that a calling mobile unit is $1500 \mathrm{~m}$ away from the base station receiver, and the mobile unit causing the interference is $50 \mathrm{~m}$ away from the base station receiver. Then the Signal-to-Interference ratio at the base station receiver with mean path loss $d^{-4}$ is [4], [5]

$$
\frac{S}{I}=10 \log \left(\frac{1500}{50}\right)^{-4}=-59.08 d B
$$

For reliable communication an $\frac{S}{I}$ of $18 \mathrm{~dB}$ is suffcient. This means that to completely avoid the ACI, 
the filter must have at least $(-55.08-18=)-77.08 \mathrm{~dB}$ attenuation in the stop band to filter out the near-endfar-end interference. Assuming the filter of channel B has a roll off $24 \mathrm{~dB} /$ octave, a frequency separation of is required.

$$
\frac{B}{2} 2^{\frac{-S / I}{24}}=4.632 B \approx 5 \text { channels }
$$

\section{Channel Assignment Algorithms}

We consider the DCA schemes [1], [2], and [3] which incorporate the CI constraint, for our work. These are (i)FA (First Available) [1]

In FA, the first available channel with in the reuse distance encountered during a channel search is assigned to the call.

(ii)LODA (Locally Optimized Dynamic Assignment) [2]

In the LODA strategy the selected cost function is based on the future blokinng probability in the vicinity of the cell in which a call is initiated.

(iii) DCA [3]

In this scheme a channel is assigned/released as given below:

- Assign the channel that is available in the smallest number of interfering cells. ...... (a)

\section{- Release}

(free) the channel that becomes available in the greatest number of interfering cells.

Then, we apply ACI constraint by defining appropriate cost function for channel allocation. We propose the following new algorithms, and demonstrate our procedure by applying it to [3].

Algorithm 1: Assign the channel that provides the required channel separation ( $r$ ) to avoid $A C I$ (Adjacent Channel Interference).

Algorithm 2 : Swap the channels if a channel with ACI constraint is not available.

Let us consider a cell $c$ where

$\operatorname{INTF}(c)$-Set of interfering cells with the cell $c$, $A V L(c)$-Set of available channels in the cell $c$, $A S S(c)$-Set of assigned channels in the cell $c$, $L_{O C K}(c h)$-Number of times the channel $c h$ is locked in cell $k$,

$N$-Total No. of duplex channels,

$R$-Cell radius,

$D$-Reuse distance $(D=\sqrt{3 K} R)$,

$K$-Cluster size $\left(K=i^{2}+i j+j^{2}\right)$.

\section{Cost Function $\mathrm{C1}_{\mathrm{c}}$}

The allocation cost contribution for the channel ch $\in$ $A V L(c)$ for the statement (a) can be expressed as

$$
C 1_{c}(c h)=\Sigma_{k}\left\{A_{k}(c h)\right\}
$$

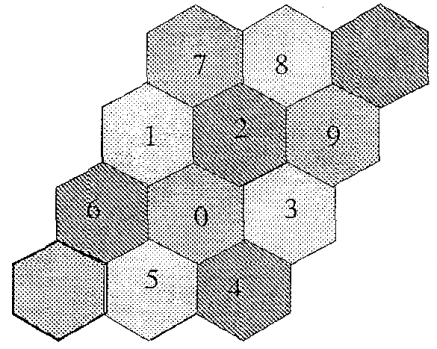

Figure 1:

where $k \in I N T F(c)$ and $A_{k}(c h)$ is given by

$$
A_{k}(c h)= \begin{cases}1 & \text { if } c h \in A V L(k) \\ 0 & \text { otherwise }\end{cases}
$$

To assign an optimal channel $c h \in A V L(c)$ in cell $c$, cost function for each channel ch $\in A V L(c)$ is calculated and the channel, $c h$, which gives minimum $C 1_{c}(c h)$ is assigned.

\section{Cost Function $\mathrm{C2}_{\mathrm{c}}$}

The cost function for the statement (b) can be expressed as

$$
C 2_{c}(c h)=\Sigma_{k}\left\{A_{k}(c h)\right\}
$$

where $k \in I N T F(c)$, ch $\in A S S(c)$ and $A_{k}(c h)$ is given by

$$
A_{k}(c h)= \begin{cases}0 & \text { if } L O C K_{k}(c h)=1 \\ 1 & \text { otherwise }\end{cases}
$$

To release a channel for maximum possible channel utilization in cell $c$, the cost function for each channel ch $\in A S S(c)$ is calculated and the channel, ch, which gives minimum $C 2_{c}(\mathrm{ch})$ is released.

\section{Cost Function $\mathrm{C3}_{\mathrm{c}}$}

The allocation cost contribution for the channel ch $\epsilon$ $A V L(c)$ for the Algorithm 1 can be expressed as

$$
C 3_{c}(c h)= \begin{cases}0 & \text { if } d(c h)>0 \\ 1 & \text { otherwise }\end{cases}
$$

where $d(c h)$ is given by

$d(c h)=\min \{|c h-u|: u \in A S S(c), \operatorname{ch} \in A V L(c)\}-r$ where $r$ : Channel Separation and from (1) \& (3) the Cost Function $\mathbf{C}_{\mathbf{c}}$ can be expressed as

$$
C_{c}(c h)=C 1_{c}(c h) C 3_{c}(c h)
$$

To assign an optimal channel ch $\in A V L(c)$ in cell $c$ with ACI constraint, overall cost function for each channel $c h \in A V L(c)$ is calculated and the channel, $c h$, which gives $C_{c}(c h)=0$ is assigned. Otherwise optimal channel is not available and the call is blocked.

To clarify how the DCA scheme operates, we refer to Fig. 1 and Example 1. For simplicity we consider the $D=3 R$ (i.e. $i=1, j=1$, and $K=\sqrt{3}$ ). 
$\operatorname{INTF}(0)=\{1,2,3,4,5,6\}$

$\operatorname{AVL}(0)=\{1,6\}, \operatorname{ASS}(0)=\{2\}$

$\operatorname{AVL}(1)=\{2,6\}, \operatorname{ASS}(1)=\{3\}$,

$\operatorname{AVL}(2)=\{2\}, \operatorname{ASS}(2)=\{5\}$,

$\operatorname{AVL}(3)=\{6\}, \operatorname{ASS}(3)=\{4\}$,

$\operatorname{AVL}(4)=\{1,5,6\}, \operatorname{ASS}(4)=\{7\}$,

$\operatorname{AVL}(5)=\{1,3,4,6\}, \operatorname{ASS}(5)=\{\}$,

$\operatorname{AVL}(6)=\{4,6\}, \operatorname{ASS}(6)=\{5\}$,

\section{Example 1:}

Let us consider that a call arrives in cell $c=0$ as shown in the Fig.1. For this cell, we have $\operatorname{INTF}(c)=$ $\{1,2,3,4,5,6\}, A V L(c)=\{1,6\}, A S S(c)=\{2\}$ then we calculate the cost for each channel $c h \in A V L(c)$ using the equation (1)

$$
\begin{aligned}
& C 1_{c}(1)=\sum_{k=1}^{6} A_{k}(1)=2 \\
& C 1_{c}(6)=\sum_{k=1}^{6} A_{k}(6)=5
\end{aligned}
$$

Therefore, the optimal channel 2 is assigned.

Applying the same Example 1 to the statement (3), and calculating the cost for each channel ch $\in A V L(c)$, using the equation (4)

$$
\begin{gathered}
d(1)=-4<0 \quad C 3_{c}(1)=1 \\
d(6)=-1<0 \quad C 3_{c}(6)=1 \\
C_{c}(1)=C 1_{c}(1) C 3_{c}(1)=2 \\
C_{c}(6)=C 1_{c}(6) C 3_{c}(6)=5
\end{gathered}
$$

Therefore, optimal channel (i.e. which gives $\left.C_{c}(c h)=0\right)$ is not available and that call is blocked.

According to the algorithm 1 , although there are available channels $(A V L(0) \neq\{\phi\})$ in cell ' 0 ', the ACI constraint prevented the allocation of a channel and the call is blocked. In order to overcome this problem we propose the algorithm Channel Swapping based Dynamic Channel Allocation (CS-DCA). This is described in the following section.

\section{CS-DCA}

To swap the channels $\operatorname{ch} 1 \in A S S(c), \operatorname{ch} 2 \in$ $A S S(k)$, such that to assign the channel ch $\epsilon$ $A V L(c)$ in cell $c$, the operations involved are as follows

(i) The channel ch1 should not have been assigned in any of the interfering cells of cell $k$, except in cell $c$. This is represented by swap constraint

$$
\begin{array}{r}
S_{\mathrm{ck}}(\operatorname{ch} 1)= \begin{cases}0 & \text { if } \operatorname{ch} 1 \notin\{A S S(j)\} \\
1 & \text { otherwise }\end{cases} \\
\text { where } j \in \operatorname{INTF}(k), j \neq c
\end{array}
$$

\begin{tabular}{|c|c|c|c|c|}
\hline$\overline{\mathrm{DCA}}$ & $\mathrm{w} / \mathrm{o}$ & with & After & Percentage \\
\hline Scheme & $\mathrm{ACI}$ & $\mathrm{ACI}$ & Swapping & improvement \\
\hline Efa & 6.85 & 5.60 & 6.30 & 12.50 \\
\hline Eloda & 7.25 & 5.85 & 6.45 & 10.26 \\
\hline E_dca & 8.15 & 6.25 & 6.80 & 8.80 \\
\hline C_fa & 6.25 & 5.20 & 5.75 & 10.58 \\
\hline C_loda & 6.25 & 5.20 & 5.75 & 10.58 \\
\hline C_dca & 7.50 & 6.05 & 6.50 & 7.44 \\
\hline
\end{tabular}

- (ii) Similarly the channel ch2 should not have been assigned in any of the interfering cells of cell $c$, except in cell $k$. Which is represented by

$$
\begin{gathered}
S_{k c}(\operatorname{ch} 2)=\left\{\begin{array}{lc}
0 & \text { if } \operatorname{ch} 2 \notin\{A S S(j)\} \\
1 & \text { otherwise }
\end{array}\right. \\
\text { where } j \in \operatorname{INTF}(c), j \neq k
\end{gathered}
$$

Table 1: For a GOS of 5\% the offered traffic in Erlangs for different schemes and percentage improvement in traffic

- (iii) The channel $c h 1$ should fallow the required ACI constraint in cell $k$. It is represented by

where

$$
d_{c k}(\operatorname{ch} 1)>0
$$

$$
d_{c k}(\operatorname{ch} 1)=\min \{|\operatorname{ch} 1-i|: i \in A S S(k), i \neq \operatorname{ch} 2\}-r
$$

- (iv) The channel ch2 should fallow the required ACI constraint in cell $c$, and the channel ch 2 should also fallow the required channel separation with the channel $c h$ in cell $c$. For this

$$
\begin{gathered}
d 1_{k c}(\operatorname{ch} 2)>0 \\
d 2_{k c}(\operatorname{ch} 2)>0
\end{gathered}
$$

where

$$
\begin{aligned}
& d 1_{k c}(\operatorname{ch} 2)=\min \{|\operatorname{ch} 2-j|: j \in A S S(c), j \neq c h 1\}-r \\
& d 2_{k c}(\operatorname{ch} 2)=|\operatorname{ch} 2-\operatorname{ch}|-r
\end{aligned}
$$

and from (iii) \& (iv) the Frequency Separation Constraint is represented by

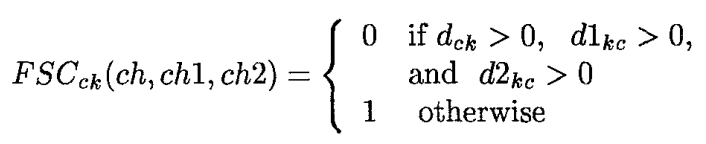

\section{Overall Cost Function $\mathrm{C}_{\mathrm{c}} \mathrm{k}$}

The overall cost function for the channel swapping is expressed as

$$
\begin{gathered}
C_{c k}(\operatorname{ch}, \operatorname{ch} 1, \operatorname{ch} 2)=S_{c k}(\operatorname{ch} 1)+S_{k c}(\operatorname{ch} 2) \\
+F S C_{c k}(\operatorname{ch}, \operatorname{ch} 1, \operatorname{ch} 2)
\end{gathered}
$$

If the cost function $C_{c k}(\operatorname{ch}, \operatorname{ch} 1, \operatorname{ch} 2)=0$, the channels $\operatorname{ch} 1, \operatorname{ch} 2$ are swappable such that the channel $c h$ can be assigned in cell $c$.

Let us consider the same Example.1, i.e., a call arrives in cell $c=0$, and the cell has $\operatorname{INTF(c)=}$ $\{1,2,3,4,5,6\}, A V L(0)=\{1,6\}, A S S(0)=\{2\}$ then we calculate the cost for each channel $\operatorname{ch} \in A V L(c)$

$$
\begin{gathered}
S_{c k}(2)=0 \quad S_{k c}(7)=0 \\
\text { where } c=0, k=4 \\
d_{c k}(2)>0, d 1_{k c}(7)>0, d 2_{k c}(7)>0 \\
F S C_{c} k(1,2,7)=0 \\
C_{c k}(1,2,7)=0
\end{gathered}
$$

Therefore, the channel 2 of cell 0 is swappable with the channel 7 of cell 4 such that the channel 1 of cell 0 can be assigned to the new call. 


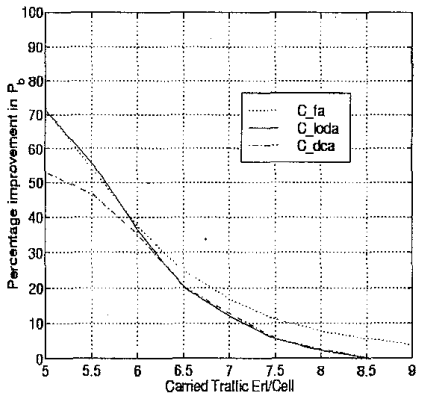

Figure 2: Percentage improvement vs Offered traffic with Center cells

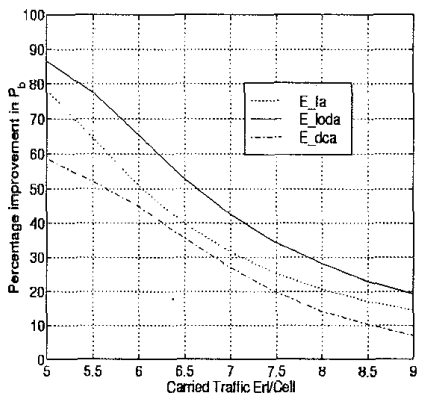

Figure 3: Percentage improvement vs Offered traffic with Edge cells

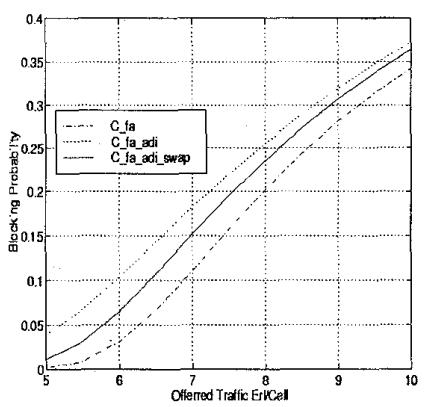

Figure 4: $P_{b}$ vs Offered traffic (FA with Center cells

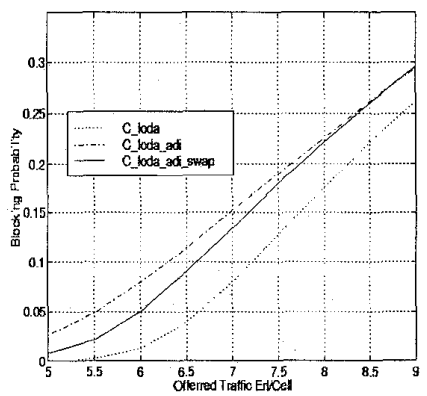

Figure 5: $P_{b}$ vs Offered traffic (LODA with Center cells)

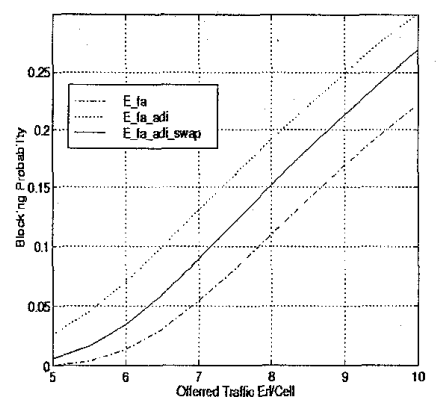

Figure 6: $P_{b}$ vs Offered traffic (FA with Edge cells)

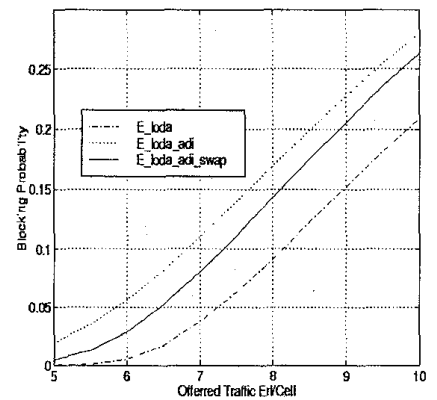

Figure 7: $P_{b}$ vs Offered traffic (LODA with Edge cells)

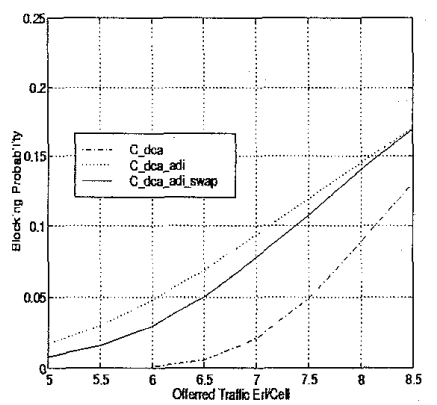

Figure 8: $P_{b}$ vs Offered traffic (DCA with Center cells)

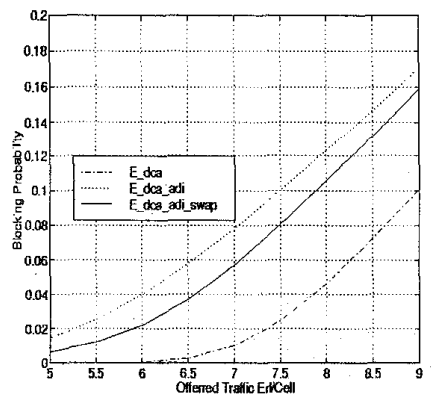

Figure 9: $P_{b}$ vs Offered traffic (DCA with Edge cells) 


\section{Performance Evaluation}

Performance evaluation of the proposed scheme has been carried out using the following model on DCA schemes [1], [2], and [3].

\section{SIMULATION MODEL}

The cellular layout is hexagonal with cell radius 1500 m. Total number of cells $10 \times 10: 100$ and full duplex channels available at every base station are 70 . The call arrival process is poisson and the call duration (channel holding time) is exponential. Calls are blocked only if there is no channel available at call setup and there is no call dropping. Receiver filter has fall off characteristics of $-24 \mathrm{~dB} /$ octave. The simulation is interference limited with $S / I$ of $18 \mathrm{~dB}$, and frequency separation of 5 channels. Propagation model: Mean path loss $d^{-4}$, Log normal shadowing with standard deviation $\sigma=6.5 \mathrm{~dB}$ and the reuse distance $D=\sqrt{3 K} R$ where $\mathrm{K}$ (cluster size) $=7$ is considered.

\section{RESU̇LTS AND DISCUSSIONS}

The newly proposed CS-DCA scheme is simulated using programming language $\mathrm{C}++$ on Ultra Sparc workstations, and its performance is evaluated with DCA schemes explained in [1], [2], and [3] in terms of call blocking and offered traffic.

Performance of CS-DCA for Edge cells (for which the set of interfering cells is incomplete) and Center cells (for which the set of interfering cells is complete) are shown in Fig. 2-9, where the term "E_" on the plots represents the results for "Edge cells", "C_" represents for "Center cells", "adi" represents the results with ACI (Adjacent Channel Interference Constraint), and "swap" represents the results of newly proposed scheme.

For a GOS (Grade of Service) of $5 \%$ the improvement in the offered traffic for different schemes is shown in Table 1. The new Channel Swapping based Dynamic Channel Allocation scheme performs better for low traffic as shown in Fig. 2 and Fig. 3. Further Comparing the results for Edge cell with Center cells in figures, it is found that the improvement in the offered traffic for Edge cells is higher than the Center cells.

Thus, the results clearly show that the new scheme gives a considerable improvement in the offered traffic. However, this improvement in performance is achieved at the cost of additional computational complexity.

\section{Conclusion}

The Channel Swapping based Dynamic Channel Allocation scheme presented in this paper improves offered traffic by swapping the assigned channels from the interfering cells to assign a channel which is about to be blocked because of the ACI constraint. Our simulation results show that the traffic carrying capacity can be increased by about $10 \%$ for GOS of $5 \%$, and this clearly shows the usefulness of the proposed scheme in modern cellular communication systems.

\section{References}

[1] D. C. Cox and D. O. Rendink, "DCA in Two Dimension Large-Scale Mobile Radio System," Bell Sys. Technol., Vol.51, 1972, pp. 1611-28.

[2] M. Zhang and T. S. Yum, "Comparisons of channel assignment strategies in cellular mobile telephone system", IEEE Trans. Veh. Technol., Vol.38, pp. 211-215, Nov. 1989.

[3] E. Del Re, R. Fantacci "Handover and Dynamic Channel Allocation Techniques in Mobile Cellular Networks," IEEE Trans. on Veh. Technol., Vol. 44, No. 2, May 1995.

[4] William C. Y. Lee, Mobile Cellular Telecommunications, McGraw-Hill, 1995.

[5] Theodore S. Rappaport, Wireless communications, Prentice Hall, 1996.

[6] J. Chuang, "Autonomous adaptive frequency assignment for TDMA portable radio systems," IEEE Trans. Veh. Technol., pp. 627-635, Aug. 1991.

[7] M. Cheng, "Distributed measurement-based quasifixed frequency assignment for personal communications," in Proc. IEEE ICC'95, Seattle, June 1995, pp. 433-437.

[8] S. H. Oh et al., "Prioritized Channel Assignment in a Cellular Radio Network," IEEE Trans. on Commun., Vol. 40, 1992 pp. 1259-69.

[9] S. Golestaneh, "The Effect of Adjacent Channel Interference on the Capacity of FDMA Cellular Systems," IEEE Trans. Veh. Technol. Vol. 43, No. 4, Nov. 1994.

[10] N. Kinoshita, "Method of rejecting adjacent channel interference using an adaptive equalizer," Electron. Commun. in Japan, part 1, Vol. 72, no. 11, 1989. 